\title{
Annexin A2 functions downstream of c-Jun N-terminal kinase to promote skin fibroblast cell migration
}

\author{
YOUPEI WANG ${ }^{1 *}$, XINMEI WU ${ }^{2 *}$, QING WANG $^{3}$, MEIQIN ZHENG $^{1}$ and LINGXIA PANG ${ }^{3}$ \\ ${ }^{1}$ Clinical Examination Center, The Affiliated Eye Hospital of Wenzhou Medical University, Wenzhou; \\ ${ }^{2}$ The Second Affiliated Hospital of Wenzhou Medical University, Wenzhou, Zhejiang 325000; \\ ${ }^{3}$ Function Experiment Teaching Center of Wenzhou Medical University, Wenzhou, Zhejiang 325035, P.R. China
}

Received January 31, 2016; Accepted February 1, 2017

DOI: $10.3892 / \mathrm{mmr} .2017 .6535$

\begin{abstract}
Delayed healing of skin wounds is one of the outcomes of diabetes mellitus (DM), a condition that affects a significant number of patients worldwide. However, the underlying mechanisms remain unknown. In order to examine proteome alterations in DM, a rat model of type 1 diabetes was developed using streptozotocin injections. The proteomic responses of normal and DM rat skin were analyzed by two-dimensional electrophoresis, and differentially expressed proteins were identified using a liquid chromatography/mass spectrometry system. DM induced 36 and repressed 41 differentially expressed proteins, respectively. Altered proteins were involved in a number of biological processes, including RNA and protein metabolism, the tricarboxylic acid cycle, glycolysis, cytoskeleton regulation, hydrogen detoxification and calcium-mediated signal transduction. In addition, overexpression of annexin A2, one of the signaling proteins altered by $\mathrm{DM}$, accelerated the rate of human skin fibroblast cell migration. Application of SP600125, an inhibitor of a key regulator of cell migration c-Jun N-terminal kinase (JNK), inhibited the migration of normal cells. By contrast, SP600125 treatment did not inhibit the migration of annexin A2-overexpressed cells, indicating that annexin A2 may function downstream of JNK. In conclusion, the results of the present study reveal the potential proteomic responses to DM in skin tissues, and
\end{abstract}

Correspondence to: Professor Meiqin Zheng, Clinical Examination Center, The Affiliated Eye Hospital of Wenzhou Medical University, 268 Xueyuanxi Road, Wenzhou, Zhejiang 325000, P.R. China

E-mail: 863979778@qq.com

Professor Lingxia Pang, Function Experiment Teaching Center of Wenzhou Medical University, Xueyuanxi Road, Wenzhou, Zhejiang 325035, P.R. China

E-mail: 10445966@qq.com

*Contributed equally

Key words: proteomic, cell migration, skin, diabetes, annexin A2 demonstrate a positive functional role of annexin A2 in fibroblast cell migration.

\section{Introduction}

Diabetes mellitus (DM) is one of the most severe metabolic diseases, which affects $>170$ million people worldwide (1). A major symptom of DM is an increased blood sugar level, which results in a number of additional complications, such as skin ulcers, which affect $\sim 15 \%$ of DM patients (1). In addition, DM affects angiogenesis, which leads to the delay of wound healing (2); however, the underlying mechanisms remain unclear.

Wound healing is a complex process that requires the cooperation of keratinocytes, fibroblasts, endothelial cells, macrophages and platelets (3). Fibroblast cell proliferation and migration is known to serve an important role in the wound repair process, as well as in collagen deposition and remodeling, wound contraction, angiogenesis and the formation of extracellular matrix (ECM) (3). It is possible that poor DM-associated wound healing may be due to altered protein and lipid metabolism and the abnormal formation of granulation tissue (4). Increased blood sugar levels alter glycosylation enzymes and induce covalent binding of aldose sugars to a protein or lipid. Binding of aldose sugar to a protein or lipid produces a stable product, termed advanced glycation end-product, which accumulate on the surface of the cell membrane and ECM proteins leading to delayed turnover rates (4). Reactive oxygen species (ROS) accumulate in high quantities in the skin of diabetic rats (5). In addition, nitric oxide (NO) is a known signaling molecule that stimulates fibroblast cell proliferation, maturation, differentiation and collagen production during the wound healing process (6). In fibroblasts from diabetic ulcers, the cells often possess a dilated endoplasmic reticulum, an increased number of vesicular bodies and defected micro tubular structures (7). These subcellular alterations may be induced by activated protein synthesis and turnover, as well as defects in microtubule structure thus inhibiting protein secretion via autonomous trafficking through vesicles and induction of microtubules to release their products into the extracellular space in diabetic ulcer fibroblasts $(7,8)$. Diabetic ulcers exhibit defective fibroblast proliferation, which may be due to reduced ECM protein production, and delayed wound contraction and 
healing (8). However, a limited number of biochemical studies have been performed to date, that have investigated the mechanisms underlying DM-mediated delay of wound healing (5-8).

Fibroblast cell migration is an important step during the wound closure process, and the phosphoinositide 3-kinase (PI3K)-Ras-related C3 botulinum toxin substrate 1-c-Jun N-terminal kinase (JNK) signaling pathway is important in the promotion of cell migration (9). In a recent study, RNA-Seq analysis was performed using high-glucose stimulated human foreskin fibroblasts, in order to examine the response of a large population of transcripts to high glucose-induced stress (9). The results demonstrated that the inflammatory response and Wnt signaling pathways generated negative and positive responses to high glucose-induced stress, respectively (10). Xuan et al (10) revealed that nuclear factor- $\kappa \mathrm{B}$ functions independently of PI3K signaling to activate JNK, resulting in accelerated fibroblast cell migration.

The present study investigated DM-induced alterations in a large number of proteins in diabetic rat skin using two-dimensional (2-D) gel electrophoresis. Proteomic analysis and liquid chromatography/mass spectrometry (LC/MS) were performed to identify the differentially expressed proteins. In addition, the effect of annexin A2 on human foreskin fibroblast cell migration was analyzed, as well as the potential association between annexin A2 and JNK, a key regulator of cell migration. The present study provides useful proteomic information that may enhance what is currently known regarding the mechanisms underlying skin wound closure.

\section{Materials and methods}

Generation of skin wounds in diabetic rats. A rat model of type 1 diabetes was generated using a previously described method (11). A total of 12 male Sprague-Dawley rats (age, 8 weeks; weight, 150 to $200 \mathrm{~g})$ were housed in cages $(12 \mathrm{~h}$ light $/ 12 \mathrm{~h}$ dark cycle; free access to water and food) at $25^{\circ} \mathrm{C}$ and $30 \%$ humidity, divided into 2 groups ( $\mathrm{n}=6 /$ group) and were injected intraperitoneally with streptozotocin (STZ; Sigma-Aldrich; Merck KGaA, Darmstadt, Germany; 50 mg/kg) which was dissolved in sodium citrate buffer ( $\mathrm{pH} 4.5)$ (12). STZ-injected rats with blood glucose levels $>16.7 \mathrm{mmol} / 1$ were considered to have developed DM. To generate the control group, the same volume of sodium citrate without STZ was injected into the male rats. To generate skin wounds, the rats were anesthetized with pentobarbital (Sigma-Aldrich; Merck $\mathrm{KGaA} ; 45 \mathrm{mg} / \mathrm{ml})$, and two circular wounds $\left(\sim 200 \mathrm{~mm}^{2}\right)$ that were $1 \mathrm{~cm}$ deep were created on the lower back of each rat. Ethical approval was obtained from the ethics committee of Wenzhou Medical University (Wenzhou, China).

Analysis of wound healing. Following 8 weeks of DM onset, the wound area was evaluated every 4 days for 16 days. The skin wounds from 3 diabetic and 3 control rats were photographed with rulers, and the area of the unhealed regions was calculated using these images together with TINA 2.0 software (DesignSoft, Inc., Budapest, Hungary; http://www. designsoftware.com/) (11).

Protein preparation and 2-D SDS-PAGE analysis. Following anesthesia with pentobarbital $(45 \mathrm{mg} / \mathrm{ml}$; Sigma-Aldrich;
Merck $\mathrm{KGaA}$ ), the dorsal area of diabetic rats was totally depilated with $\mathrm{Na}_{2} \mathrm{~S}(8.0 \%$, w/v; Sigma-Aldrich; Merck $\mathrm{KGaA})$ and two full-thickness circular wounds $\left(\sim 300 \mathrm{~mm}^{2}\right.$ each) were created on the lower back of each rat using a pair of sharp scissors and a scalpel. Skin tissues ( $2 \mathrm{~g})$ that were 1-cm deep were collected from the diabetic and control rats for protein extraction 2 weeks following the establishment of DM. The following reagents were used for protein extraction: PBS, $9.5 \mathrm{M}$ urea, $0.1 \%$ (w/v) DTT, $2 \%$ (w/v) CHAPS and $0.8 \%$ (w/v) pharmalyte (Sigma-Aldrich; Merck KGaA). The extracts were dissolved in immobilized $\mathrm{pH}$ gradient (IPG) rehydration buffer [8 M urea, 2\% (w/v) 3-[(3-cholamidopropyl) dimethylammonio]-1-propanesulfonate hydrate, $2 \%(\mathrm{w} / \mathrm{v})$ IPG buffer, $0.04 \mathrm{M}$ dithiothreitol, 1X nuclease solution and $0.1 \%(\mathrm{w} / \mathrm{v})$ of bromophenol blue] prior to isoelectric focusing electrophoresis. The following reagents were used for 2-D gel analysis: 1\% DTT, $50 \mathrm{mM}$ Tris-HCl, pH 8.8, $6 \mathrm{M}$ urea, 30\% (v/v) glycerol, $2 \%$ (w/v) SDS, $0.1 \%$ (w/v) bromophenol blue, equilibration buffer $(2.5 \%$ (w/v) iodoacetamide (Sigma-Aldrich; Merck KGaA). The MS/MS spectra were processed using Proteome Discoverer software (version 1.3; Thermo Fisher Scientific, Inc., Waltham, MA, USA) and the database search was performed using the Mascot search engine (Matrix Science Mascot version 2.3; http://www.matrixscience.com/) against a concatenated forward-decoy approach (13).

In-gel digestion. Protein spots were visualized using ImageMaster 2D Platinum version 6.0 (GE Healthcare Bio-Sciences, Pittsburgh, PA, USA). A total of 77 protein spots of interested were excised and digested using a $1 \%$ protease cocktail blue (Sigma-Aldrich; Merck KGaA), which were further analysed using MS. In-gel digestion was performed in 3 steps according to the procedure described by Russell et al (14). The following reagents were used for analysis: $50 \%$ (v/v) acetonitrile (ACN), 10 mM DTT containing $100 \mathrm{mM}$ ammonium bicarbonate and $1 \%$ trypsin solution (Sigma-Aldrich; Merck KGaA).

$L C-M S / M S$ analysis and bioinformatics analysis. The proteins extracted from the gel sections were digested using $1 \%$ trypsin solution (Sigma-Aldrich; Merck KGaA) and $4 \mu \mathrm{l}$ of samples were submitted to online nanoflow liquid chromatography using the easy-nano LC system (Proxeon Biosystems; Thermo Fisher Scientific, Inc.) with $10 \mathrm{~cm}$ capillary columns of an internal diameter of $75-\mu \mathrm{m}$, filled with 3- $\mu \mathrm{m}$ Reprosil-Pur C18-A2 resin (Dr. Maisch GmbH, Ammerbuch-Entringen, Germany) (14). The gradient consisted of $10-30 \%$ (v/v) ACN in $0.1 \%(\mathrm{v} / \mathrm{v})$ formic acid at a flow rate of $200 \mathrm{nl} / \mathrm{min}$ for $45 \mathrm{~min}, 30-100 \%(\mathrm{v} / \mathrm{v}) \mathrm{ACN}$ in $0.1 \%(\mathrm{v} / \mathrm{v})$ formic acid at a flow rate of $200 \mathrm{nl} / \mathrm{min}$ for $1 \mathrm{~min}$ and $100 \% \mathrm{CAN}$ in $0.1 \%$ formic acid at a flow rate of $200 \mathrm{nl} / \mathrm{min}$ for $10 \mathrm{~min}$. The elution was electrosprayed through a Proxeon nanoelectrospray ion source by (electrospray ionization) ESI-MS/MS analysis on a Thermo Fisher LTQ Velos Pro (Thermo Fisher Scientific, Inc.) using full ion scan mode over the $\mathrm{m} / \mathrm{z}$ range 200-1800. Collision-induced dissociation (CID) was performed in the linear ion trap using a 4.0-Th isolation width and 35\% normalized collision energy with helium as the collision gas. Five independent MS/MS scans were performed on each ion using dynamic exclusion. The precursor ion that was selected for 
CID was dynamically excluded from further MS/MS analysis for $30 \mathrm{sec}$. Further corresponding protein identification was performed using the Swiss-Prot protein sequence database (version 54.5; http://web.expasy.org/docs/swiss-prot_guideline.html). LC-MS/MS and bioinformatics analyses were performed by LC Bio, Inc. (Hangzhou, China).

Western blot analysis. Skin tissues $(100 \mathrm{mg})$ were ground using liquid nitrogen and an ice-cold lysis solution (7 M urea, $2 \mathrm{M}$ thiourea, 2\% CHAPS, $40 \mathrm{mM}$ Tris base, $40 \mathrm{mM}$ dithiothreitol, and $1 \%$ protease inhibitor; Sigma-Aldrich; Merck KGaA) was added to procure whole cell extracts. Total proteins $(20 \mu \mathrm{g})$ from skin samples with or without induced diabetes 2 weeks prior, were separated on a $12 \%$ SDS-PAGE gel and electrotransferred to Immobilon ${ }^{\circledR}$-P PVDF Transfer Membranes (EMD Millipore, Billerica, MA, USA). The membranes were blocked in $1 \mathrm{X}$ Tris-buffered saline containing 5\% skim milk and $0.05 \%$ Tween-20 for $2 \mathrm{~h}$. All of the reagents for SDS gel electrophoresis and membrane blocking were purchased the Sigma-Aldrich; Merck KGaA. To analyse protein levels in the samples the membranes were incubated with the following primary antibodies at room temperature $\left(25^{\circ} \mathrm{C}\right)$ for 2 h: Anti-eNOS antibody (cat. no. ab76198; 1:2,000, Abcam, Cambridge, MA, USA), anti-cleaved (c)-caspase-3 antibody (cat. no. ab2302; 1:2,000, Abcam), anti-plasminogen activator inhibitor (PAI) -1 antibody (cat. no. ab125687; 1:2,000; Abcam), anti-cluster of differentiation (CD) 34 antibody (cat. no. ab81289; 1:2,000; Abcam), anti-collagen1 antibody (cat. no. ab34710; 1:2,000; Abcam), anti-annexin A2 antibody (cat. no. ab41803; 1:1,000; Abcam), anti-JNK antibody (cat. no. ab179461; 1:2,000; Abcam), anti-phosphorylated (p) -JNK antibody (cat. no. ab4821; 1:2,000; Abcam) and anti-GAPDH antibody (cat. no. ab8245; 1:2,000; Abcam). The membranes were then incubated with either the anti-mouse or anti-rabbit horseradish peroxidase-conjugated secondary antibody (cat. no. 7074; 1:2,000; Cell Signaling Technology, Inc., Danvers, MA,USA) at room temperature $\left(25^{\circ} \mathrm{C}\right)$ for $2 \mathrm{~h}$. Antigen-antibody complexes were visualized using an electrochemiluminescence kit (BioTrand, Crystal Lake, Illinois, USA). Protein levels were normalized against GAPDH and protein expression was analyzed using Image J2 version 2.0 software (13).

Human foreskin fibroblast cell culture. Human fibroblast cell culture was performed as previously described (5). Human foreskin tissues were collected from 3 patients diagnosed with redundant prepuce (all three patients were healthy without DM or other disease diagnoses; ages, 26, 30 and 32 years old) who were admitted in January 2014 to the Department of Dermatology at the First Affiliated Hospital of Wenzhou Medical University (Wenzhou, China). The present study was approved by the ethics committee of Wenzhou Medical University, and written informed consent was obtained from all patients involved. The fat was first removed from the tissue samples, when were subsequently cut into $3 \times 2-m m$ strips and incubated overnight at $4^{\circ} \mathrm{C}$ in $0.05 \%$ Dispase I (Sigma-Aldrich; Merck KGaA). The epidermis was then removed and the dermis was placed into $25 \mathrm{~cm}^{2}$ flasks pre-treated with foetal bovine serum (FBS; HyClone; GE Healthcare Life Sciences, Logan, UT, USA). The flasks were positioned horizontally for $1 \mathrm{~h}$, and then vertically for $3 \mathrm{~h}$ in a culture chamber at $37^{\circ} \mathrm{C}$ with $5 \% \mathrm{CO}_{2}$. The cells were cultured in Dulbecco's modified Eagle's medium (DMEM; HyClone; GE Healthcare Life Sciences) containing $5.5 \mathrm{mM}$ glucose (Sigma-Aldrich; Merck KGaA), 10\% FBS and 1\% penicillin-streptomycin (Gibco; Thermo Fisher Scientific, Inc.); the medium was refreshed every 3 days. When cell confluence had reached 70 to $80 \%$ the cells were digested and passaged using $0.25 \%$ trypsin (Gibco; Thermo Fisher Scientific, Inc.). Cells were cultured for 3 days in $5.5 \mathrm{mM}$ glucose medium, before they were transferred to media with or without $25 \mu \mathrm{M}$ SP600125 (Cell Signaling Technology, Inc.) for up to 1 day prior to the migration assay, or for $1 \mathrm{~h}$ prior to western blot analysis.

Overexpression of annexin A2 in fibroblast cells. For overexpression of annexin A2, the open reading frame (ORF) sequences of the annexin A2 gene (ANXA2; GenBank Accession no. NM_001002858.2; National Center for Biotechnology Information, https:/www.ncbi.nlm.nih.gov/) was amplified for 28 cycles of $95^{\circ} \mathrm{C}$ for $30 \mathrm{sec}, 60^{\circ} \mathrm{C}$ for $30 \mathrm{sec}$ and $72^{\circ} \mathrm{C}$ for $90 \mathrm{sec}$ using the following primers: Forward, 5'-ATCGTAGGATCCATGGGCCGCCAGCTAG-3', and reverse, 5'-AGCTATTCTAGATCAGTCATCTCCACCACA CAGGTA-3'. The amplified polymerase chain reaction (PCR) fragments were then cloned into the Bacillus amyloliquefaciens and Xanthomonas badrii restriction enzyme sites of the pcDNA3 expression vector (Invitrogen; Thermo Fisher Scientific,Inc.). Primer design and sequencing were performed by Sangon Biotech Co., Ltd. (Shanghai, China; http://www. sangon.com/). A total of 2 or $3 \mu \mathrm{g}$ pcDNA3-ANXA2 plasmid was transformed into human foreskin primary fibroblasts from the 3 patients using a Lipofectamine 2000 kit (Invitrogen; Thermo Fisher Scientific, Inc.) according the manufacturer's instructions. Empty vector pcDNA3 using Lipofectamine 2000 transformed cells was used as control. The 2 and $3 \mu \mathrm{g}$ of pcDNA3-ANXA2 plasmid transformed cells were termed OX1 and OX2, respectively. Following 2 days of transformation, annexin A2 levels in the fibroblast cells were examined by western blotting, and cell migration tests were performed.

Cell migration assay. Primary fibroblast cells were seeded onto 6-well plates at $80-90 \%$ confluency and incubated at $37^{\circ} \mathrm{C}$ overnight in DMEM containing $0.5 \% \mathrm{FBS}$ and $5 \mu \mathrm{g} / \mathrm{ml}$ mitomycin-C (Sigma-Aldrich; Merck KGaA). Linear scratch wounds were subsequently created in the confluent fibroblast monolayer using a sterile $200 \mu \mathrm{l}$ pipette tip (Ningbo MFLab Medical Instruments Co., Ltd., Ningbo, Zhejiang, China). The medium was immediately replaced with prewarmed $\left(37^{\circ} \mathrm{C}\right)$ fresh DMEM containing $0.5 \% \mathrm{FBS}$ and $5 \mu \mathrm{g} / \mathrm{ml}$ mitomycin-C. At 0,12 and $24 \mathrm{~h}$ following wound establishment, images were captured using a light microscope (IX70; Olympus Corporation, Tokyo, Japan) equipped with a CCD camera (CoolSNAP HQ; Nippon Roper, Toyko, Japan), which was controlled by MetaMorph 7.1 software (Molecular Devices, LLC, Sunnyvale, CA, USA). To quantify cell migration, 10 cells on the border of the wound area were randomly selected from each well and the migration distance was measured using ImageJ version 14.8 software (https://imagej.nih.gov/ij/) at the indicated time points. 
Statistical analysis. Statistical analyses were performed using GraphPad Prism software (version 5.0; GraphPad Software, Inc., La Jolla, CA, USA). Comparisons between 2 groups were performed using a Student's t-test, while significant differences among 3 groups were analysed using one-way analysis of variance, followed by Bonferroni's multiple comparison test. $\mathrm{P}<0.05$ was considered to indicate a statistically significant difference.

\section{Results}

DM impairs skin wound healing. Diabetes-induced skin wound healing was analyzed in a rat model of diabetes. STZ, an inducer of type 1 diabetes was used to induce diabetes in rats, and wound healing was assessed by monitoring and comparing the size of $200 \mathrm{~mm}^{2}$ circular wounds created on the waist of each rat at 8 weeks following the onset of diabetes (8). Wound repair was then analysed over the course of 16 days, and the results indicated that the rate of wound healing was significantly reduced in diabetic rats when compared with non-diabetic normal rats (Fig. 1).

Proteomic analysis of rat skin tissues under DM stress. In order to gain an improved understanding of the molecular mechanisms underlying DM-mediated impairment of skin wound healing, comparative proteomic assays were preformed to identify differentially expressed proteins in the skin of DM rats and normal rats using 2-D gel electrophoresis. Total skin protein extracts were first subjected to 2-D gel analysis followed by identification by LC/MS. This led to the detection of proteins altered by DM. Proteins extracted from the normal rat skin were used as a control (Fig. 2). Optimization of the 2 -D gels and image processing identified 36 and 41 protein spots (representing differentially expressed proteins) that were induced and repressed by DM, respectively (Fig. 2). The experiments were repeated 3 times and only the reproducible alterations were taken into account. To identify differentially expressed proteins, the spots were excised from the 2-D gels and identified by LC/MS and trypsin digestion. The results presented in Table I demonstrate that 72 proteins were identified among the 77 spots observed in the 2-D gel (Fig. 2). The major group of proteins identified, either overexpressed or repressed, were observed to be involved in regulation of the cytoskeleton (tubulin and actin formation), carbohydrate metabolism (glycolysis and the tricarboxylic acid cycle) and protein folding. In the present study, the calcium binding proteins, calmodulin and calreticulin, were induced in DM (Table I). Formation of the ECM is an important step in the wound healing process (3). DM reduced the levels of collagen $\alpha 1$ and dermatopontin, two ECM-associated proteins. In addition to the major group of proteins, RNA metabolism, adenosine triphosphate (ATP) synthesis and cell migration-associated protein levels were altered (Table I).

Protein expression was altered in DM rat skin. To verify the results identified by proteomic assay, western blot analysis was performed using specific antibodies to detect select marker proteins in the pathways identified. The results revealed that the expression levels of CD34, collagen-1 and endothelial NO synthase were decreased (NO synthesis), whereas PAI-1

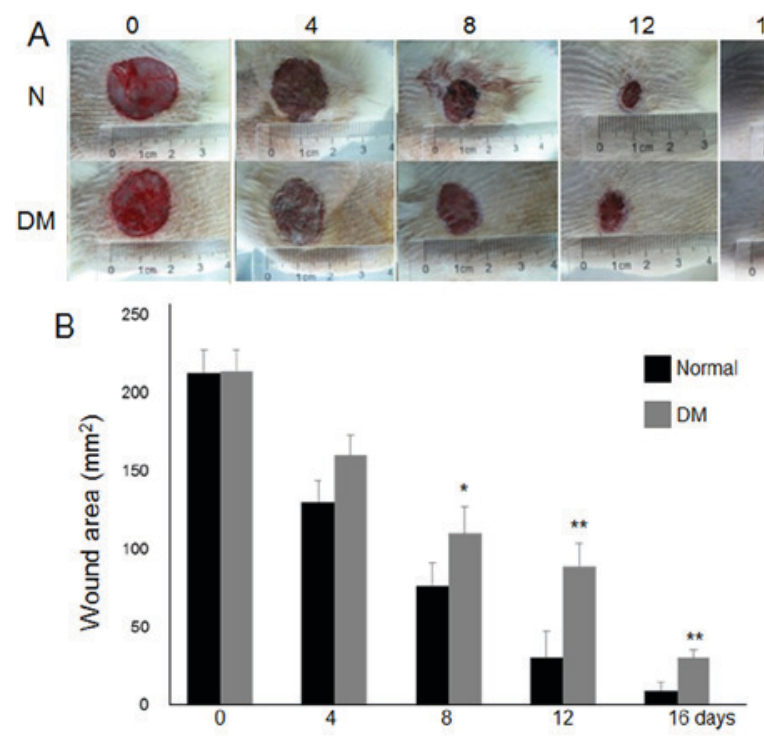

Figure 1. Effect of DM on the rate of skin wound repair in rats. (A) Representative images of skin wounds from normal and DM rats. (B) The wound area in normal and DM rats at each time point. Wound areas were measured using TINA 2.0 software. ${ }^{*} \mathrm{P}<0.05$ and ${ }^{* *} \mathrm{P}<0.05$ vs. control. DM, diabetes mellitus; N, normal group.

(inflammation), c-caspase 3 (apoptosis) and annexin A2 were increased in DM rat skin when compared with normal rat skin (Fig. 3). These results were similar to those identified by the proteomic analysis.

Overexpression of annexin A2 promotes human foreskin fibroblast cell migration. Annexin A2 was induced in DM rat skin (Fig. 3), and the transcript of its orthologue gene in humans was previously demonstrated to be induced by high-glucose treatment of foreskin fibroblast cells (9). Therefore, the function of annexin A2 in fibroblast cells was examined further, by forced overexpression of annexin A2. Human ANXA2 (GenBank Accession no. NM_001002858.2) ORF sequences were amplified by PCR, and the PCR products were then cloned into the pcDNA3 expression vector. Following sequencing, 2 and $3 \mu \mathrm{g}$ pcDNA3-ANXA2 plasmid were transformed into human foreskin primary fibroblast cells using the Lipofectamine 2000 system to generate two independent transformants, termed OX1 and OX2, respectively. Compared with the control cells (untransformed), OX1 and OX2 cells exhibited a significantly increased cell migration rate following wound generation (Fig. 4A and B). To verify the expression levels of annexin A2, western blot analysis was performed. The results demonstrated that the level of annexin A2 was increased in OX1 and OX2 when compared with the controls (Fig. 4C). In addition, annexin A2 overexpression maintained an increased cell migration rate when compared with the control cells when they were cultured in high-glucose containing medium (data not shown). However, the level of p-JNK, a key regulator of cell migration, was not altered by the overexpression of annexin A2 (Fig. 4C).

Annexin A2 functions downstream of JNK. As annexin A2 overexpression was not observed to alter p-JNK protein 
Table I. Differentially expressed proteins identified by liquid chromatography/mass spectrometry analysis.

A, Proteins highly expressed in DM skin

\begin{tabular}{|c|c|c|c|}
\hline Spot no. & UniProt ID & Protein & Function \\
\hline 1 & P69897 & Tubulin $\beta-5$ chain & Cytoskeleton \\
\hline 8 & P85108 & Tubulin $\beta$-2A chain & Cytoskeleton \\
\hline 9 & Q4QRB4 & Tubulin $\beta$ & Cytoskeleton \\
\hline 13 & P48675 & Desmin & Cytoskeleton \\
\hline 16 & O35763 & Moesin & Cytoskeleton \\
\hline 17 & O35763 & Moesin & Cytoskeleton \\
\hline 2 & P18418 & Calreticulin & $\mathrm{Ca}^{2+}$ binding \\
\hline 4 & P18418 & Calreticulin & $\mathrm{Ca}^{2+}$ binding \\
\hline 12 & P43742 & Reticulocalbin-3 & $\mathrm{Ca}^{2+}$ binding \\
\hline 34 & P62161 & $\begin{array}{l}\text { Calmodulin } \\
\text { (inflammation and apoptosis) }\end{array}$ & $\mathrm{Ca}^{2+}$ binding \\
\hline 3 & P06761 & $78 \mathrm{kDa}$ glucose-regulated protein & Protein folding and oligomerization \\
\hline 7 & P04785 & Protein disulfide-isomerase & Protein folding \\
\hline 19 & P11598 & Protein disulfide-isomerase A3 & Protein folding \\
\hline 36 & P11598 & Protein disulfide-isomerase A3 & Protein folding \\
\hline 24 & P34064 & Proteasome subunit $\alpha$ type- 5 & Protein degradation \\
\hline 14 & G3V9R8 & Heterogeneous nuclear ribonucleoprotein $\mathrm{C} 1 / \mathrm{C} 2$ & RNA metabolism \\
\hline 6 & P61980 & Heterogeneous nuclear ribonucleoprotein $\mathrm{K}$ & RNA metabolism \\
\hline 22 & B5DEN5 & Elongation factor $1-\beta$ & RNA metabolism \\
\hline 33 & $\mathrm{P} 02401$ & $60 \mathrm{~S}$ acidic ribosomal protein $\mathrm{P} 2$ & RNA metabolism \\
\hline 35 & P63324 & 40S ribosomal protein $\mathrm{S} 12$ & RNA metabolism \\
\hline 20 & P62630 & Elongation factor $1-\alpha 1$ & RNA metabolism \\
\hline 5 & P33840 & Antithrombin-III & Plasma protease inhibitors \\
\hline 10 & P10719 & ATP synthase subunit $\beta$ & ATP synthesis \\
\hline 11 & P31000 & Vimentin & Signaling \\
\hline 23 & P20332 & 14-3-3 protein $\sigma$ & Signaling \\
\hline 25 & Q79342 & 14-3-3 protein $\sigma$ & Signaling \\
\hline 15 & Q9WTT6 & Guanine deaminase & Purine metabolism \\
\hline 18 & P04764 & $\alpha$-enolase & Carbon metabolism \\
\hline 21 & $\mathrm{P} 02650$ & Apolipoprotein E & Carbon metabolism (inflammation) \\
\hline 29 & P04797 & Glyceraldehyde-3-phosphate dehydrogenase & Carbon metabolism \\
\hline 30 & P04797 & Glyceraldehyde-3-phosphate dehydrogenase & Carbon metabolism \\
\hline 31 & P04797 & Glyceraldehyde-3-phosphate dehydrogenase & Carbon metabolism \\
\hline 26 & Q07936 & Annexin A2 & Apoptosis and inflammation \\
\hline 27 & P63029 & Translationally-controlled tumor protein & Tumor protein \\
\hline 28 & P23764 & Glutathione peroxidase 3 & Superoxide \\
\hline 32 & Q04374 & $\operatorname{Ig} \kappa$ chain $\mathrm{V}$-II region $26-10$ & Immune system \\
\hline
\end{tabular}

B, Proteins with reduced expression in DM skin

\begin{tabular}{llll}
\hline 37 & P02454 & Collagen $\alpha-1$ & Tissue connection \\
68 & B2R777 & Dermatopontin & ECM matrix \\
38 & P04785 & Protein disulfide-isomerase & Protein folding \\
40 & P04785 & Protein disulfide-isomerase & Protein folding \\
77 & P73842 & Peptidyl-prolylcis-trans isomerase A & Protein refolding \\
65 & P40112 & Proteasome subunit $\beta$ type-3 & Protein degradation \\
42 & P39423 & Lamin-A/C & Gene regulation \\
47 & P70564 & Serpin B5 & Gene regulation \\
61 & Q9WUH4 & Four and a half LIM domains protein 1 & Signaling \\
44 & P60711 & Actin, cytoplasmic 1 & Cytoskeleton
\end{tabular}


Table I. Continued.

B, Proteins with reduced expression in DM skin

\begin{tabular}{|c|c|c|c|}
\hline Spot no. & UniProt ID & Protein & Function \\
\hline 48 & Q4FZT0 & Stomatin-like protein 2 & Cytoskeleton \\
\hline 49 & Q6AYC4 & Macrophage-capping protein & Cytoskeleton \\
\hline 71 & Q4KLF8 & Actin-related protein $2 / 3$ complex subunit 5 & Cytoskeleton \\
\hline 66 & P02600 & Myosin light chain $1 / 3$ & Cytoskeleton \\
\hline 67 & P13413 & Troponin I & Cytoskeleton \\
\hline 72 & P45592 & Cofilin-1 & Cytoskeleton \\
\hline 73 & P04466 & Myosin regulatory light chain 2 & Cytoskeleton \\
\hline 74 & P16409 & Myosin regulatory light chain & Cytoskeleton \\
\hline 45 & P15429 & $\beta$-enolase & Carbon metabolism \\
\hline 46 & P05065 & Fructose-bisphosphate aldolase A & Carbon metabolism \\
\hline 43 & P04764 & $\alpha$-enolase & Carbon metabolism \\
\hline 50 & P12007 & Isovaleryl-CoA dehydrogenase & Carbon metabolism \\
\hline 51 & P16617 & Phosphoglycerate kinase 1 & Carbon metabolism \\
\hline 52 & P41562 & Isocitrate dehydrogenase & Carbon metabolism \\
\hline 53 & P17764 & Acetyl-CoA acetyltransferase & Carbon metabolism \\
\hline 55 & P05065 & Fructose-bisphosphate aldolase A & Carbon metabolism \\
\hline 56 & P07943 & Aldose reductase & Carbon metabolism \\
\hline 57 & P04797 & Glyceraldehyde-3-phosphate dehydrogenase & Carbon metabolism \\
\hline 58 & P04636 & Malate dehydrogenase & Carbon metabolism \\
\hline 62 & P15429 & $\beta$-enolase & Carbon metabolism \\
\hline 69 & P39069 & Adenylate kinase isoenzyme 1 & ATP synthesis \\
\hline 59 & $\mathrm{~A} 7 \mathrm{VJC} 2$ & Heterogeneous nuclear ribonucleoproteins A2/B1 & RNA metabolism \\
\hline 60 & P55260 & Annexin A4 & Apoptosis and inflammation \\
\hline 63 & P63029 & Translationally-controlled tumor protein & Tumor protein \\
\hline 64 & A48593 & Glutathione S-transferase P1 & Superoxide \\
\hline 75 & P02625 & Parvalbumin $\alpha$ & $\mathrm{Ca}^{2+}$-binding \\
\hline
\end{tabular}

Differentially expressed proteins were identified following optimization of two-dimensional gels and image processing. The spot number refers to the corresponding protein on the gel images presented in Fig. 2. DM, diabetes mellitus; ID, identification number; ATP, adenosine triphosphate, ECM, extracellular matrix.

expression levels, SP600125, an inhibitor of JNK was used to analyze its effect on annexin A2 expression. SP600125 significantly inhibited fibroblast cell migration (Fig. 5A and $\mathrm{B})$, which is consistent with results presented in a previous study (11). In addition, SP600125 suppressed p-JNK expression while maintaining normal total JNK expression levels (Fig. 5C). SP600125 treatment marginally repressed annexin A2 protein expression levels (Fig. 5C).

Due to the observed inhibitory effects of SP600125 on cell migration and annexin A2 expression levels, its effects were further examined in OX1 fibroblast cells overexpressing $A N X A 2$. SP600125+OX1 was associated with a significant increase in cell migration when compared with SP600125-treated controls at 12 and $24 \mathrm{~h}$. In addition, SP600125 treatment did not alter the expression levels of annexin A2 in OX1 transformed cells (Fig. 6A and B). Western blotting results demonstrated that SP600125 markedly suppressed p-JNK levels in OX1 cells (Fig. 6C). Collectively, these results indicate that annexin A2 may function downstream of JNK to promote fibroblast cell migration.

\section{Discussion}

One of the strategies employed by living organisms to adapt to environmental alterations, is the rapid reprogramming of transcriptional and translational regulation via cell signalling mechanisms. Therefore, the analysis of proteomic alterations following exposure to specific stressors is a suitable strategy to understand its regulatory basis $(9,10)$. In the present study, proteomic analysis was performed on DM rat skin to identify global alterations in the proteome. The proteomic results demonstrated that the expression of a number of important proteins involved in metabolic processes was significantly altered.

Cell migration, a key process in wound healing, is multi-step process. During the migration process a protrusion extends from one cell to attach to a nearby leading edge 
$(\mathrm{kDa})$

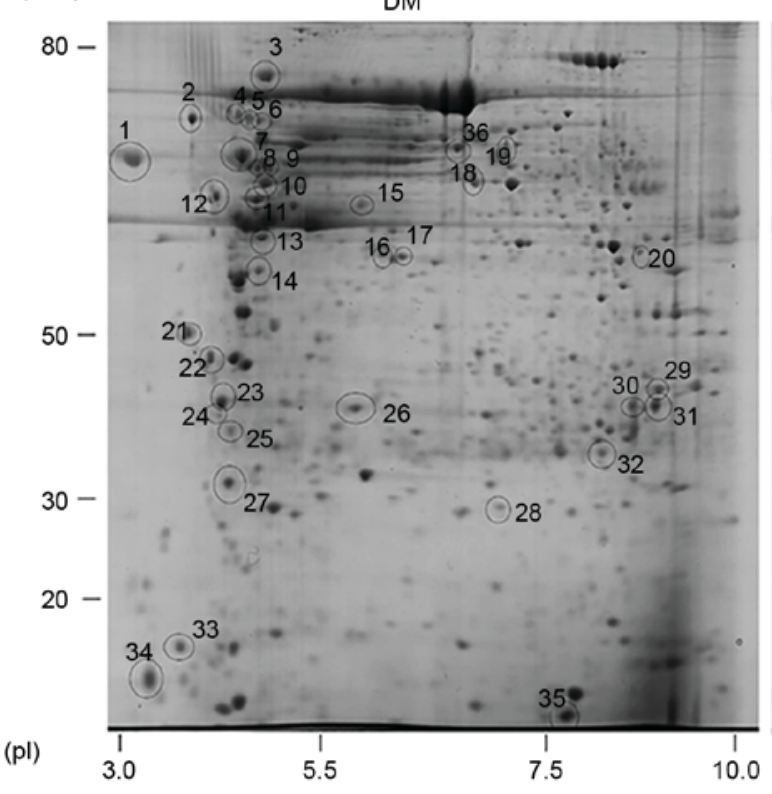

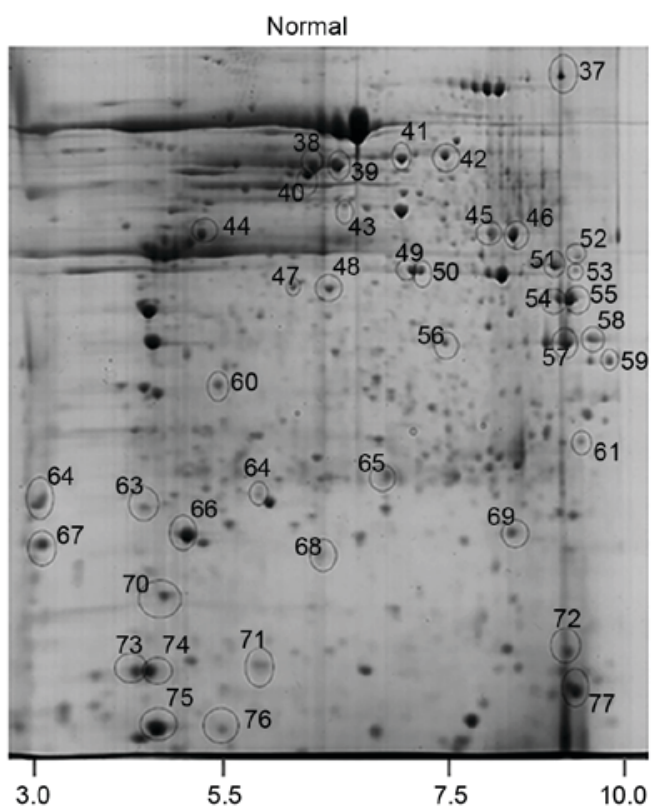

Figure 2. Comparison of 2-D gel images of the total protein extracts from normal and DM rat skins. Total protein extracted from the normal rat (right panel) and DM rat (left panel) skins were separated using a 2-D gel system and detected by silver staining. The 77 differentially expressed proteins identified in the normal and DM rat skins are indicated by circles and a corresponding number. 2-D, two-dimensional; DM, diabetes mellitus; pI, isoelectric point.

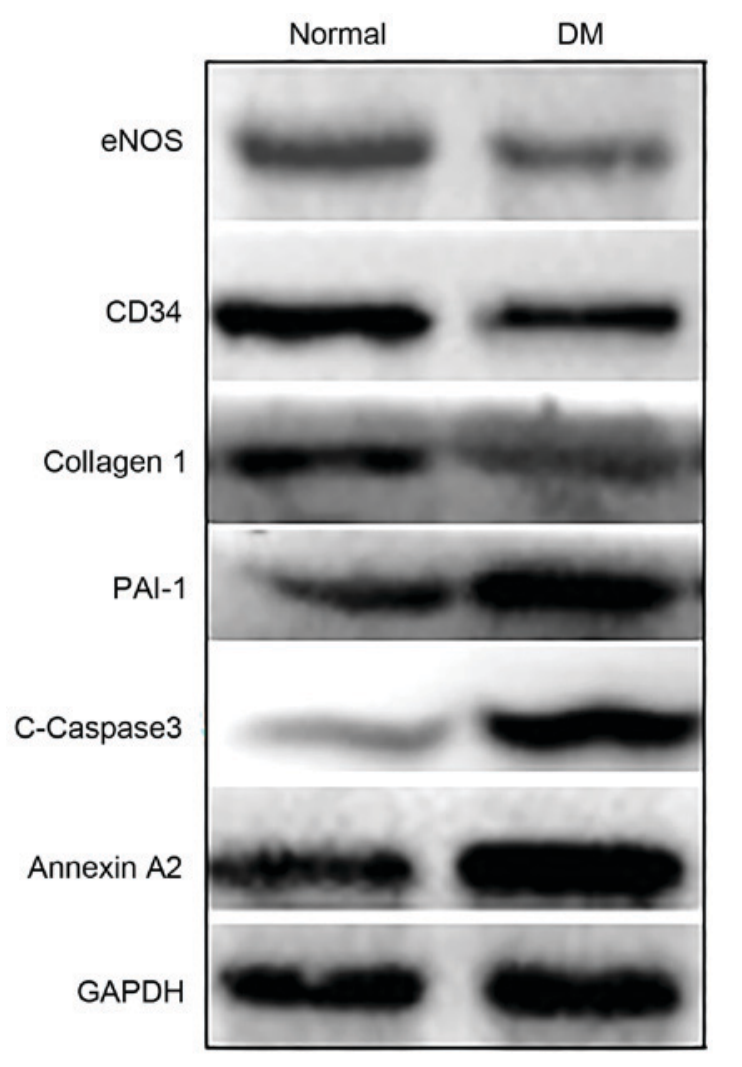

Figure 3. Western blot analysis of protein marker expression. Proteins extracted from the skin of normal and DM rats were used to analyse the expression of eNOS, CD34, annexin A2, collagen1, c-caspase-3 and PAI-1. GAPDH was used as a loading control. DM, diabetes mellitus; eNOS, endothelial nitric oxide synthase; CD34, cluster of differentiation 34; c-caspase-3, cleaved caspase-3; PAI-1, plasminogen activator inhibitor.

of another cell protrusion (3). The cells then migrate forward and release their adhesions and retractions at the rear of the

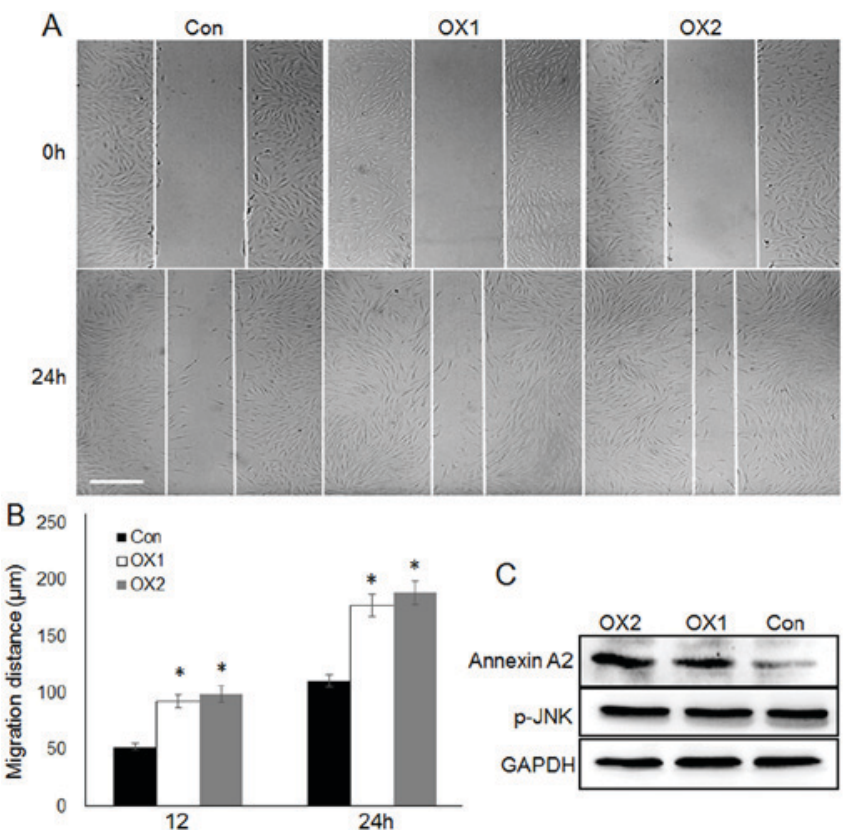

Figure 4. Effect of annexin A2 overexpression on human fibroblast cell migration. (A) A wound healing assay was performed to analyse the effects of annexin A2 overexpression on fibroblast cell migration (scale bar, $500 \mu \mathrm{m}$ ). OX1 and OX2 represent human fibroblast cells transformed with 2 and $3 \mu \mathrm{g}$ pcDNA3-ANXA2 plasmids, respectively. (B) Quantification of cell migration distance. Data represent the mean \pm standard error of 10 replicates. Significant differences between non-transformed and annexin A2-overexpressed cells are indicated; "P $<0.01$ vs. control. (C) Annexin A2 and p-JNK levels were analysed by western blot analysis. GAPDH was used as a loading control. ANXA2, annexin A2 gene; p-JNK, phosphorylated-c-Jun N-terminal kinase; Con, untransformed control cells.

cell (15-17). The actin-mediated Wiskott-Aldrich syndrome protein-family verprolin homologous protein complex is important in the formation of the lamellipodial protrusions at 
A

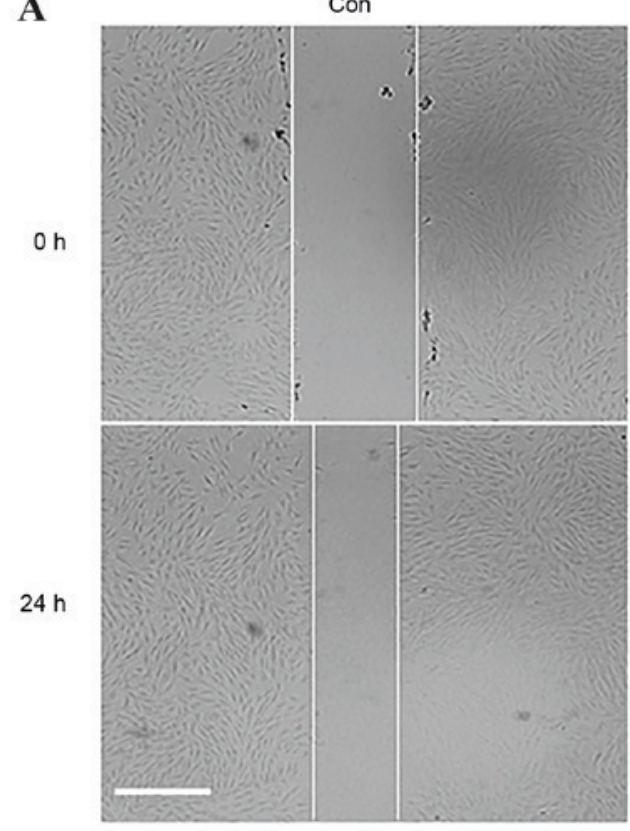

B



SP600125

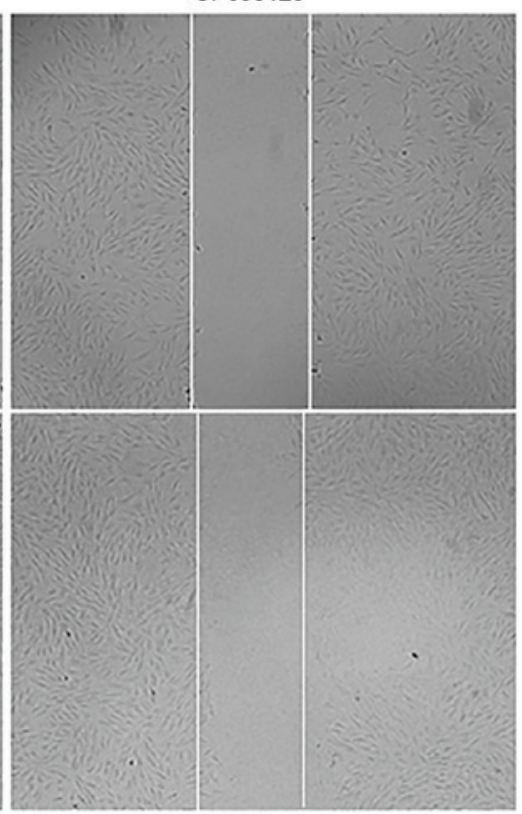

C

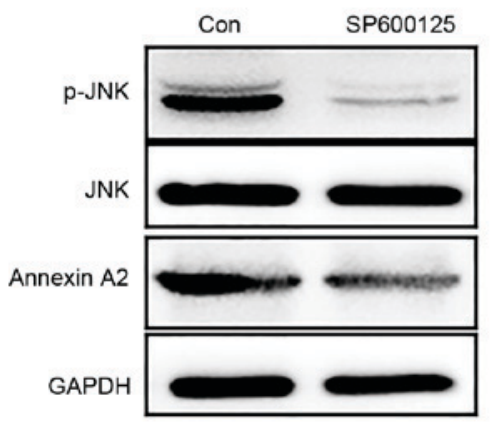

Figure 5. Effect of SP600125 on fibroblast migration and annexin A2 levels. (A) A wound healing assay was performed to analyse the effects of SP600125, an inhibitor of p-JNK, on fibroblast migration (scale bar, $500 \mu \mathrm{m}$ ). (B) Quantification of cell migration distance. (A) Data represent the mean \pm standard error of 10 replicates. The significant differences between SP600125-treated and untreated cells are indicated; ${ }^{* *} \mathrm{P}<0.001$ vs. control. (C) p-JNK, JNK and annexin A2 levels were analysed by western blot analysis. GAPDH was used as a loading control. p-JNK, phosphorylated-c-Jun N-terminal kinase; Con, untreated control cells.

the leading edge of the migrating cells (18), and the Rho family guanosine triphosphatase protein, cell division control protein 42 , reportedly regulates the polarity of migrating cells (19). In the present study, cytoskeleton-associated proteins were part of a major group of proteins regulated by DM in rat skin. Actin and the actin-associated protein complex were repressed by DM, as were tubulin, myosin, troponin and additional cytoskeleton-associated proteins. The ECM serves important roles in cell adhesion, communication between nearby cells, and during differentiation induced by the deposition of extracellular molecules secreted by cells (20). In the present study, collagen $\alpha 1$ and dermatopontin, an ECM-associated protein, were repressed in DM while moesin, a protein involved in cell-to-cell recognition, was induced. A previous study identified protein alterations induced by DM that involved the superoxide detoxification process (glutathione peroxidase 3 and glutathione S-transferase P1) and ROS accumulation in rat skin (5), indicating that DM-mediated ROS accumulation may be induced via inhibition of the superoxide detoxification pathway. In addition, DM regulation in carbon metabolism and the ATP synthesis process may affect the supply of carbon skeletons and energy to the cell, which may in turn inhibit wound closure (21).

Annexin A2, a member of the annexin protein family, has been reported to induce cell migration and neoangiogenesis in breast tumors (22). In addition, annexin A2 reportedly regulates intestinal epithelial cell spreading and wound closure (23). In the present study, annexin A2 was overexpressed while annexin A4 was repressed in DM. Western blot analysis confirmed the increased levels of annexin A2 in DM rat skin when compared with normal controls. Similarly, a previous transcriptomics study revealed the induction of ANXA2 by high-glucose in human foreskin fibroblast cells (9). Overexpression of ANXA2 in human foreskin primary 

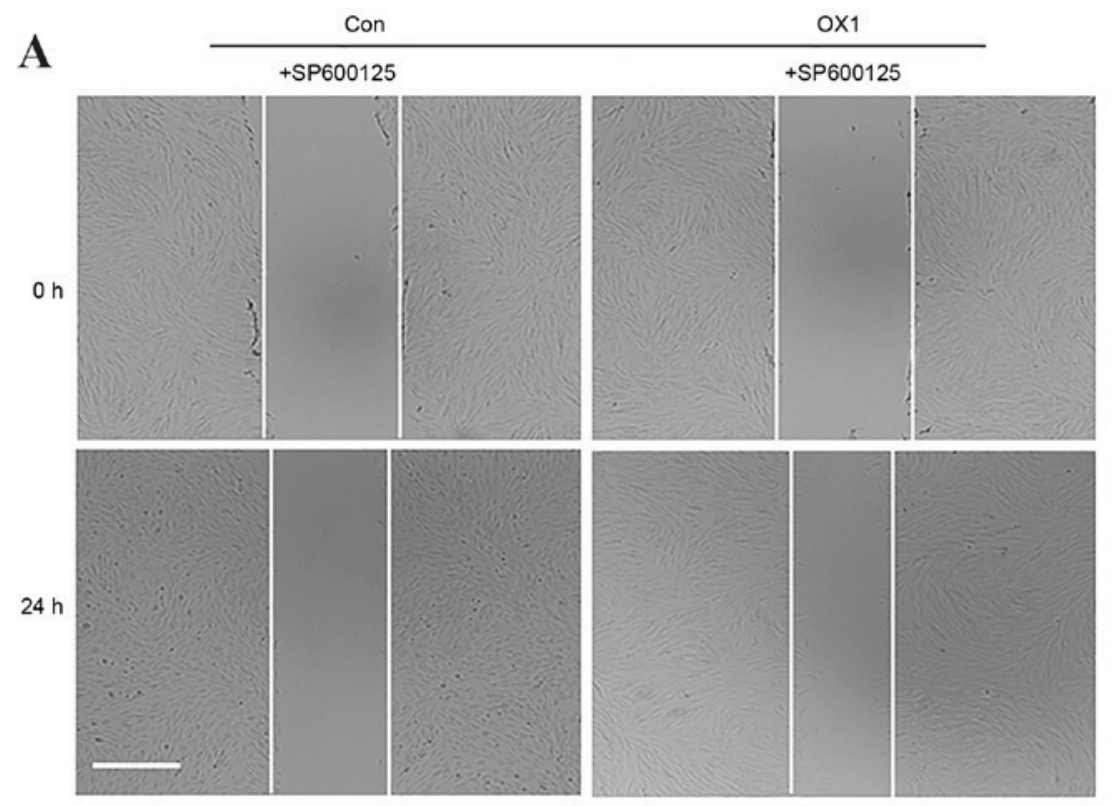

B

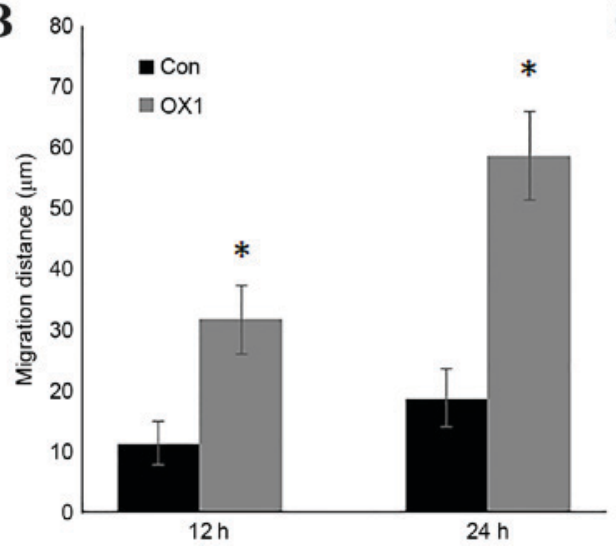

C

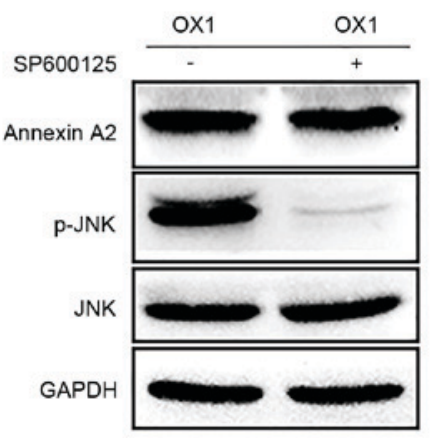

Figure 6. Effect of SP600125 on migration of cells overexpressing annexin A2. (A) A wound healing assay was performed to analyse the effects of SP600125, an inhibitor of p-JNK, on control and cells overexpressing annexin A2 (scale bar, $500 \mu \mathrm{m}$ ). (B) Quantification of cell migration distance. Data represent the mean \pm standard error of 10 replicates. Significant differences between SP600125-treated and untreated cells are indicated; ${ }^{*}<<0.05$ vs. control. (C) Annexin A2, p-JNK and JNK levels were analysed by western blot analysis. GAPDH was used as a loading control. p-JNK, phosphorylated-c-Jun N-terminal kinase; Con, untreated control cells; OX1, human fibroblast cells overexpressing annexin A2.

fibroblast cells in the present study resulted in a marked increase in the rate of cell migration. Furthermore, inhibition of JNK by treating cells with SP600125, delayed cell migration and reduced annexin A2 levels. However, a significant difference in cell migration was identified when comparing the SP600126-treated OX1 cells with the SP600126-treated control cells (Fig. 6), indicating that annexin A2 may function downstream of JNK during cell migration. A previous study demonstrated that high-glucose stress increased the nitrosative modification of annexin A2 in human foreskin fibroblasts, and that regulation occurred downstream of JNK regulation (5). These results indicated that JNK may regulate ANXA2 expression and its post-translational modifications, which may be important for wound repair. In the present study, the level of annexin A2 was increased in rat DM skin when compared with normal skin. This may be due to feedback activation of annexin A2 in an attempt to try to repair the wound in DM rat skin.

In conclusion, 2-D gel electrophoresis and LC/MS proteomics were performed to gain insight into the intricate mechanisms underlying the response to DM-mediated delay of wound healing in rats. This approach resulted in the identification of a number of differentially expressed proteins in DM that were associated with major processes, including carbohydrate metabolism, protein folding and cell redox homeostasis. The majority of proteomic studies conducted to date, that have used stress models, have been performed in rat skin against diabetes. Overexpression of annexin A2 accelerated the rate of fibroblast migration, and JNK-annexin A2 was identified as a potential pathway that promotes cell migration during wound healing. It would be of interest to analyze skin wound repair in an annexin A2 overexpressed animal model in future studies.

\section{Acknowledgements}

The present study was made possible by an initiative grant (grant no. QA2576) from Wenzhou Medical University. The authors would like to thank the undergraduate students from Wenzhou Medical University for their assistance with the experiments. 


\section{References}

1. Yach D, Stuckler D and Brownell KD: Epidemiologic and economic consequences of the global epidemics of obesity and diabetes. Nat Med 12: 62-66, 2006.

2. Braiman-Wiksman L, Solomonik I, Spira R and Tennenbaum T: Novel insights into wound healing sequence of events. Toxicol Pathol 35: 767-779, 2007.

3. Brem H and Tomic-Canic M: Cellular and molecular basis of wound healing in diabetes. J Clin Invest 117: 1219-1222, 2007.

4. Goldin A, Beckman JA, Schmidt AM and Creager MA: Advanced glycation end products: Sparking the development of diabetic vascular injury. Circulation 114: 597-605, 2006.

5. Xuan YH, Huang BB, Tian HS, Chi LS, Duan YM, Wang X, Zhu ZX, Cai WH, Zhu YT, Wei TM, et al: High-glucose inhibits human fibroblast cell migration in wound healing via repression of bFGF-regulating JNK phosphorylation. PLoS One 9: e108182, 2014

6. Obayashi K, Akamatsu H, Okano Y, Matsunaga K and Masaki H: Exogenous nitric oxide enhances the synthesis of type I collagen and heat shock protein 47 by normal human dermal fibroblasts. J Dermatol Sci 41: 121-126, 2006.

7. Rowe DW, Starman BJ, Fujimoto WY and Williams RH Abnormalities in proliferation and protein synthesis in skin fibroblast cultures from patients with diabetes mellitus. Diabetes 26: 284-290, 1977.

8. Loots MA, Lamme EN, Mekkes JR, Bos JD and Middelkoop E: Cultured fibroblasts from chronic diabetic wounds on the lower extremity (non-insulin-dependent diabetes mellitus) show disturbed proliferation. Arch Dermatol Res 291: 93-99, 1999.

9. Pang L, Wang Y, Zheng M, Wang Q, Lin H, Zhang L and Wu L: Transcriptomic study of high-glucose effects on human skin fibroblast cells. Mol Med Rep 13: 2627-2634, 2016.

10. Xuan Y, Chi L, Tian H, Cai W, Sun C, Wang T, Zhou X, Shao M, Zhu Y, Niu C, et al: The activation of the NF- $\kappa B-J N K$ pathway is independent of the PI3K-Rac1-JNK pathway involved in the bFGF-regulated human fibroblast cell migration. J Dermatol Sci 82: 28-37, 2016.

11. Yang Y, Xia T, Zhi W, Wei L, Weng J, Zhang C and Li X: Promotion of skin regeneration in diabetic rats by electrospun core-sheath fibers loaded with basic fibroblast growth factor. Biomaterials 32: 4243-4254, 2011.
12. Cai L, Wang Y, Zhou G, Chen T, Song Y, Li X and Kang YJ: Attenuation by metallothionein of early cardiac cell death via suppression of mitochondrial oxidative stress results in a prevention of diabetic cardiomyopathy. J Am Coll Cardiol 48: 1688-1697, 2006

13. Ni MW, Ye WJ, Cong WT, Hong GY, Zhu ZX, Duan YM, Zhou X and Jin LT: Fluorescent staining of protein in sodium dodecyl sulfate polyacrylamide gels by salicylaldehyde azine. Electrophoresis 34: 3171-3179, 2013.

14. Russell WK, Park ZY and Russell DH: Proteolysis in mixed organic-aqueous solvent systems: Applications for peptide mass mapping using mass spectrometry. Anal Chem 73: 2682-2685, 2001.

15. Lauffenburger DA and Horwitz AF: Cell migration: A physically integrated molecular process. Cell 84: 359-369, 1996.

16. Webb DJ, Parsons JT and Horwitz AF: Adhesion assembly, disassembly and turnover in migrating cells-over and over and over again. Nat Cell Biol 4: E97-E100, 2002.

17. Ridley AJ, Schwartz MA, Burridge K, Firtel RA, Ginsberg MH, Borisy G, Parsons JT and Horwitz AR: Cell migration: Integrating signals from front to back. Science 302: 1704-1709, 2003.

18. Smith LG and Li R: Actin polymerization: Riding the wave. Curr Biol 14: R109-R111, 2004.

19. Macara IG: Parsing the polarity code. Nat Rev Mol Cell Biol 5: 220-231, 2004.

20. Abedin M and King N: Diverse evolutionary paths to cell adhesion. Trends Cell Biol 20: 734-742, 2010.

21. Cong W, Zhao T, Zhu Z, Huang B, Ma W, Wang Y, Tan Y, Chakrabarti S, Li X, Jin L and Cai L: Metallothionein prevents cardiac pathological changes in diabetes by modulating nitration and inactivation of cardiac ATP synthase. J Nutr Biochem 25: 463-474, 2014.

22. Sharma M, Ownbey RT and Sharma MC: Breast cancer cell surface annexin II induces cell migration and neoangiogenesis via tPA dependent plasmin generation. Exp Mol Pathol 88: 278-286, 2010.

23. Babbin BA, Parkos CA, Mandell KJ, Winfree LM, Laur O, Ivanov AI and Nusrat A: Annexin 2 regulates intestinal epithelial cell spreading and wound closure through Rho-related signaling. Am J Pathol 170: 951-966, 2007. 